\title{
Metal forming technology for the fabrication of seamless Superconducting radiofrequency cavities for particle accelerators
}

\author{
Vincenzo Palmieri \\ Legnaro National Laboratories, Istituto Nazionale di Fisica Nucleare and Università di Padova, Italy
}

\begin{abstract}
The world of Particle accelerators is rather unique, since in a few high-energy Physics great laboratories, such at CERN for example, there have been built the largest technological installations ever conceived by humankind. The Radiofrequency resonant cavities are the pulsing heart of an accelerator. In case of superconducting accelerators, bulk niobium cavities, able to perform accelerating gradients up to $40 \mathrm{MeV} / \mathrm{m}$, are just a jewel of modern technology. The standard fabrication technology foresees the cutting of circular blanks, their deep-drawing into half-cells, and its further joining by electron beam welding under ultra high vacuum environment that takes several hours. However, proposals such as the International Linear Collider, to which more than 900 scientists from all over the world participate, foresee the installation of 20.000 cavities. In numbers, it means the electron beam weld one by one under Ultra High Vacuum of 360,000 hemi-cells. At a cost of $500 € / \mathrm{Kg}$ of high purity Niobium, this will mean a couple of hundreds of millions of Euros only for the bare material. In this panorama it is evident that a cost reducing approach must be considered. In alternative the author has proposed a seamless and low cost fabrication method based on spinning of fully resonators. Preliminary RF tests at low temperatures have proved that high accelerating gradients are achievable and that they are not worse than those obtainable with the standard technology. Nevertheless up to when the next accelerator will be decided to be built there is still room for improvement.
\end{abstract}

\section{Introduction}

Nobody can deny that after the discovery of the electron, our society has deeply changed. The birth of electronics has ignited a real industrial revolution. If nowadays we can use computers, washing machines, Hi-Fi or even Wi-Fi, this is due to the discovery of a small particle, as the electron is. Yet, there is a plethora of discovered elementary particles, different from the electron, such as muons, pions, kaons and so forth. So, if only one would know how to use them, we could find other disciplines such as a muonics, a pionics a kaonics and so on.

In other words, thanks to the elementary particle Physics, we could expect a new world, for our future, accompanied by several parallel industrial revolutions at one time. New particles are generally, but not only, produced in laboratory by a particle accelerator. As a microscope for a biologist or a telescope for an astronomer, a particle accelerator is the most powerful instrument in the hand of physicists, who study the nature of the subatomic constituents of matter.

This is an Open Access article distributed under the terms of the Creative Commons Attribution License 4.0, which permits unrestricted use, distribution, and reproduction in any medium, provided the original work is properly cited. 


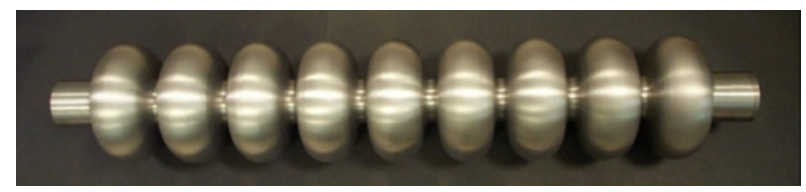

Figure 1. Photo of a 9-cell superconducting high purity $\mathrm{Nb}$ resonator for the acceleration of relativistic particles.

A particle accelerator is a device that uses electromagnetic fields to propel charged particles to high speeds and to contain them in well-defined beams. Maybe the most famous particle accelerator in the world is the Large Hadron Collider (LHC) at CERN, built into a $23 \mathrm{Km}$ of circumference ring into a tunnel 100 meters under the Geneva region in Switzerland. Other kinds of particle accelerators have been built in a large variety of applications, including particle therapy for oncological purposes, and synchrotron light sources for the study of condensed matter physics. There are currently more than 30,000 accelerators in operation around the world.

The heart of a particle accelerator is the Radio-Frequency (RF) structure, containing arrays of accelerating resonant cavities. A resonant cavity is an energy storage metallic device where a longitudinal electric field oscillate in its fundamental mode at a frequency typically ranging from $100 \mathrm{MHz}$ to around $3 \mathrm{GHz}$. If this resonant box is excited at a frequency that is synchronized with the velocity of the particle to accelerate, the particle bunch will travel as a surf on the travelling electromagnetic wave [1]. Accelerators can be based on normal conducting technology; in that case copper RF cavities are used at room temperatures. The superconducting technology, instead, is nowadays more and more common. In that case, thanks to its Critical temperature (Tc) of $9.26 \mathrm{~K}$, the highest among all simple elements in periodic table, Niobium, is used at low temperatures, typically $4.2 \mathrm{~K}$, the boiling point of liquid Helium, or also $1.8 \mathrm{~K}$, slightly under the Lambda transition, where Helium becomes superfluid implying that its thermal conductivity becomes infinite. In Fig. 1 it is possible to see a photo of a typical Niobium cavity resonant at $1.3 \mathrm{GHz}$ made of nice-cells.

The "superconducting cavity business" is a niche market, but it has a very high surplus value.

The International Linear Collider [2] for example is a proposal for a Linear Accelerator (Linac), for electron-positron collisions at energies of $500 \mathrm{GeV}$ initially, with the possibility for a later upgrade to $1 \mathrm{TeV}$. This giant accelerator would foresee 20,000 nine-cell cavities of the shape at in Fig. 1.

In parallel CERN has launched the Future Circular Collider study (FCC). With an emphasis on proton-proton and electron-positron (lepton) high-energy frontier machines, FCC proposes to explore the potential of hadron and lepton circular colliders, performing an in-depth analysis of infrastructure and operation concepts and considering the technology research and development programs that would be required to build a future circular collider [3]. In order to achieve that goal, FCC has two frontier goals: the development of Superconducting magnets and of superconducting cavities with performances close to the theoretical ones.

\section{The actual cavity fabrication technology}

Now, the Niobium utilized is high purity material, with less of $10 \mathrm{ppm}$ of tantalum; every Niobium cavity weights around $25 \mathrm{~kg}$. The current net price of Niobium in large quantities is about $600 € / \mathrm{Kg}$ plus taxes according the laws of the hosting country, plus packaging and transport expenses. Then, the total price for the whole cavity production would require a money amount of the order of 300 million Euros, only for the raw material. Such a high price does however not include any cost regarding material preparation, cavity forming, cryostats, RF electronics, vacuum pumps, magnets, all the necessary equipment for building an accelerator, including the helium liquefier, the vacuum system, magnets, particle detectors, the radio protection, securities, the building, and the personnel salaries. So, ILC is a project that could be afforded only as a joint enterprise of an international community. The reader can easily understand how 


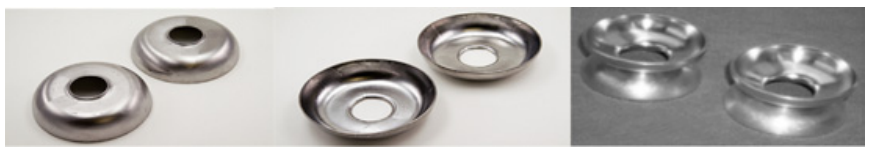

Figure 2. Nb half-cells deepdrawn from a blank and electron beam weld at the iris to form a dumbbell.

astronomical it could be the total cost of an accelerating machine project where the only raw material cost, just for only making the superconducting cavities, approaches a few hundred million Euros [4].

In theory it should be at least one possibility to drastically reduce the cost of the Niobium material: the whole cavity can be built in Oxygen Free High Conductivity (OHFC) Copper and a high purity Niobium thin film can be sputtered inside. Niobium sputtered copper cavities were invented by C. Benvenuti in early 80's at CERN for the construction of the superconducting cavities of the Large Electron Collider (LEP) [5]. Then the sputtering technology was applied in early 90's at Legnaro National Laboratories of the INFN by the author for the Quarter Wave Resonators (QWRs) of the ALPI heavy ion accelerator [6]. The $\mathrm{Nb} / \mathrm{Cu}$ technology, then, has been chosen again at CERN nowadays for the QWRs fabrication of the ISOLDE ion beam Facility [7]. Nevertheless these just above mentioned are three stories of success, the use of $\mathrm{Nb}$ sputtered Copper Cavities in particle accelerator is not as common as desired, because for thin film cavities the superconducting performances exponentially decay as a function of the RF injected power. The reason under the exponential decay of Niobium sputtered cavities is still unknown. The hypothesis formulated by the author are twofold: The transport properties of the film are not comparable with that of the bulk; the quality of the Niobium-Copper interface is quite poor, since Niobium and Copper are not miscible for any percentage in the alloy phase diagram.

In addition to the Niobium cost, the fabrication cost is not a minor problem. The standard 9-cell cavity fabrication protocol foresees: the cutting of 18 circular blanks; its deep-drawing; the half-cell calibration; the edge trimming; the further Electron beam welding of two half cells into one dumbbell and then the progressive welding to form a full nine-cell, as understandable from Fig. 2.

The bulk Niobium EB technology has been selected for the construction of the European X-Ray Free Electron Laser (X-FEL), that, starting in 2017 at the Deutches Electronen Syncrotronen (DESY) in the Hamburg area, will completely open up new research opportunities for scientists and industrial users [8]. Electrons are first brought to high energies in a superconducting accelerator. They then fly on a slalom course through a special arrangement of magnets (the "undulator"), in which they emit laser-like flashes of radiation. The first part of the facility is a 1.7-kilometre-long particle accelerator that brings bunches of electrons to high energies at nearly the speed of light. The European XFEL will generate ultrashort $\mathrm{X}$-ray flashes-27 000 times per second and with a brilliance that is a billion times higher than that of the best conventional X-ray radiation sources. X.-FEL will open up areas of research that were previously inaccessible. Using the X-ray flashes of the European XFEL, scientists will be able to map the atomic details of viruses, decipher the molecular composition of cells, take three-dimensional images of the nanoworld, film chemical reactions, and study processes such as those occurring deep inside planets. For the Superconducting Linac of X-FEL, more than a thousand Superconducting resonators have been built by the German and Italian Industry, according to the standard Protocol of Electron Beam weld bulk Niobium half-cells.

\section{The seamless forming methods}

The Cavity industrial production has shown that the mass production is possible, but also it has put in evidence the limits of the actual technology that are the excessive cost and the gap between the initially expected performance values and the achieved ones. In other words, larger accelerators will be 


\section{MATEC Web of Conferences}

never built, unless an inexpensive cavity fabrication technique will sort out from industrial research and development.

Other forming methods mainly based on the seamless forming techniques are then worthwhile to be explored.

If Niobium for example was superplastic, all our forming problems would be solved at once. Superplasticity indeed is a state in which a solid crystalline material is deformed well beyond its usual breaking point, usually over about $200 \%$ ad more, during tensile deformation. Such a state is usually achieved at high homologous temperature. Superplastically deformed material gets thinner in a uniform manner, rather than narrowing into a "neck" that leads to fracture. So, in such a case, one could dispose a circular planar blank onto a cavity-shaped hollow die, and by simply inflating a low pressure gas, the Niobium could gradually take the shape of a multicell cavity. At a more detailed analysis, it must be also noticed that in literature only a few observations of superplasticity have been reported for pure metals, and up to now, pure Niobium has not yet been found super-plastic.

Therefore, unless of electrochemical galvanoforming a thick wall cavity, in order to build a seamless cavity as the one in Fig. 1, the most evident solution is to bulge or swage a seamless tube. In this framework several techniques are possible.

One possible seamless technique would for example consist in explosive forming. This is a technique often used for forming large thick bulks of metal that are not convenient neither to machine nor to form otherwise, as for example large valve bodies for gas pipelines.

In order to fabricate a cavity by explosive forming, a seamless and uniform thickness thick tube is hosted inside a cavity-shaper hollow die. The tube is filled with water and inside a little explosive charge is wrapped into a plastic bag in the center of a cell. In order to be effective, the explosive must be a very fast one, since its power is the ratio between the released energy and the time of deflagration. Often Pentaerythritol Tetranitrate (Penthrite) is used both because it is a very fast explosive that is insoluble in water. The shock wave will propagate across the water and the cavity will be bulged. Several intermediate annealing treatments are necessary and these are costly. The explosive forming has been tried for cavity fabrication both at KEK [9] and then at LNL-IFN [10]. However, due to the intrinsic dangerousness of the technique and the cost of the intermediate annealings, the interest in a deeper investigation of the technique has generally dropped off.

The same conclusion is valid for electromagnetic forming, a type of high velocity, cold forming process for either bulging or swaging electrically conductive metals. A workpiece is reshaped by high intensity pulsed magnetic fields that induce a current onto the tube surface. The wall portion of the workpiece is then plastically deformed by the induced Lorentz forces. The tube is initially reshaped without any contact from a tool, for being then pressed against a die at the final stage of the forming process. The electromagnetic forming technique is an extremely powerful technique and it has also the advantage that Lubricants are unnecessary, so forming can be used in clean conditions. Nevertheless, the technology is costly; it requires a perfectly cylindrical tube with exceptional thickness uniformity in order to avoid wrinkling; because of the high voltages and currents involved it requires careful safety considerations.

Hydroforming is a cost-effective way of shaping ductile metals into structurally stiff and strong pieces, particularly popular with the high-end sports car and bicycle frame industry.

Good cavities have been produced instead by hydroforming by W. Singer at DESY [11, 12].

A seamless tube of intermediate diameter between cavity equator and iris is required. In a first step the tube is necked by spinning at the iris area and at the ends. In a second step, the final forming is then performed by expanding the tube in the equator area with internal water pressure.

Mono-cells and three cells cavities have been successfully hydroformed. Nine-cell resonators were even fabricated by EB welding together 3 segments of hydroformed three-cells. The reason for 3-cells is that the hydroforming machine available at DESY had size limitations. The extension to 9-cells requires significant investment and development with a 9-cell hydroforming machine. 


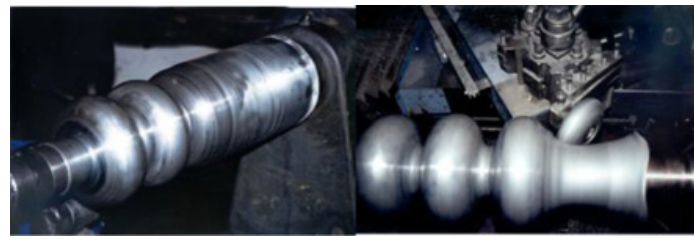

Figure 3. A seamless nine-cell resonator can be easily spun from a seamless tube. The technique is applicable both to Copper, Niobium and Niobium clad Copper bilayer.

The hydroformed cavity RF results are recently reported in literature [13]. In summary the nine-cells produced by joining the three-cell units achieve at $2 \mathrm{~K}$ a maximum accelerating field of $35 \mathrm{MV} / \mathrm{m}$, with a Q-value at the highest gradients exceeding $1 \mathrm{E}+10$. Even if these cavities were not completely weldfree in so far as they had 2 welds at the iris joining, these results are extremely interesting, because they clearly show the seamless cavity feasibility. Another advantage of Hydroforming is that also $\mathrm{Nb}$ clad copper cavities have been successfully fabricated.

Hydroforming has three minor drawbacks:

1. The hydroforming machine is good only for the cavity shaper for which it has been designed. Whenever a different cavity geometry is required, the whole machine must be re-designed.

2. The thickness uniformity of the starting tube required for hydroforming is absolutely crucial. Even a granulometry variation across the niobium tube wall could result in a different resistance of the wall portion under pressure, and a consequent fracture meanwhile bulging.

3. Only the outside shape of a cell can be precisely formed. The accuracy and reproducibility of the inside shape exposed to the RF fields (determining the frequency of the cell) depend on the wall thickness, that clearly changes during hydroforming.

A short fabrication time and surprisingly low cost technique for fabricating seamless ninecell resonators was proposed almost two decades ago by the author [10, 14-16]. The techniques originally consisted in spinning a cavity starting directly form a large blank of $\mathrm{Cu}, \mathrm{Nb}$ or $\mathrm{Nb}$ clad $\mathrm{Cu}$.

Nowadays, the spinning of seamless cavities has achieved a higher level of accuracy, and a cavity is spun, as in Fig. 3, directly from a seamless tube preliminarily produced either by deep drawing or by flowforming. For spinning, the tube does not require to have the best thickness uniformity, since meanwhile spinning, the material will be kneaded, and this is one of the main advantages of the spinning technique.

The tube is swaged by spinning onto a collapsible mandrel that has exactly the same shape of the cavity interior. In other words, the geometry of the cavity interior, i.e. the surface exposed to RF fields, is fixed by the internal die, resulting in a great accuracy for determining the resonator frequency. This is one of the most immediate advantages for the spinning technique. However, the main advantage for spinning is the significant cost reduction; almost 5 times lower that the fabrication cost according the standard U.H.V. EB welding technique.

Generally, one ninecell resonator is built in less than one working day, but it must be said that the longest part of the working time is spent setting up the machine. The whole forming operation takes less than 10 minutes per cell, which is much shorter than the time for the standard half-cell drawing, edge trimming, positioning into the EB Chamber, vacuum pumping and welding. The dismounting operation of the mandrel takes a few minutes. This means that in case of a possible industrial production, if working with dedicated machines, the fabrication times would be much shorter than the actual times. Completely seamless mono-cell, three-cells, five-cells and nine-cells have been produced at $1.3 \mathrm{GHz}$, but cavities of very different shape and frequency (from $500 \mathrm{MHZ}$ to $6 \mathrm{GHZ}$ ) can be produced always by the same spinning machine. 


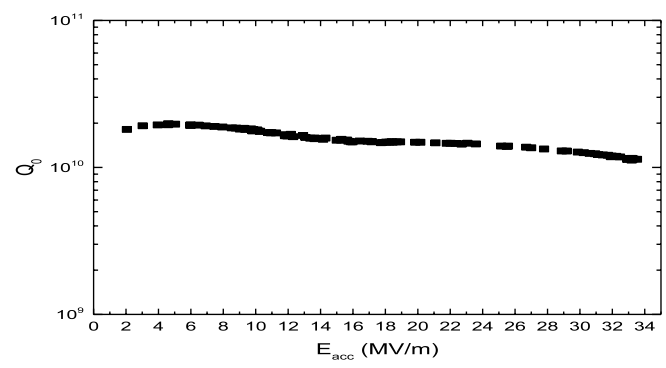

Figure 4. Excellent performance of a 3-cell spun cavity spun at LNL and tested at FNAL, after mechanical grinding, barrel finishing, vacuum annealing, electropolishing and high pressure rinsing.

After spinning, cavities must be internally tumbled or mechanically ground, then electropolished. Low temperature radiofrequency tests have proven that the seamless approach and in particular spinning is a solution worthwhile to pursue. Q-values over $1 \mathrm{E}+10$ and accelerating fields over $40 \mathrm{MV} / \mathrm{m}$ have been reproducibly measured on all the latest spun monocell prototypes, and treated and measured in different laboratories.

A seamless spun three-cell, fabricated at LNL of the INFN, has been recently measured at Fermi National Accelerator Laboratories (FNAL) in USA. The result is extremely encouraging: a Q-factor slightly lower than $2 \mathrm{E}+10$, a maximum accelerating field of $34 \mathrm{MV} / \mathrm{m}$ has been measured, clearly showing that despite the low cost of the spun cavities, experimental results that absolutely deserve consideration are obtainable.

\section{Conclusions}

Fundamental science is the key for a better life in a better world and the high energy physics done with particle accelerators can have much more practical applications than normally considered. Superconducting accelerating cavities are the heart of an accelerator and the construction of future accelerating machines will depend crucially on the possibility to build high performance RF cavities.

The standard fabrication technique based on cell deep-drawing and further EB welding, has been successfully adopted for building a masterpiece of modern accelerating technology, but it is too costly for being considered for further proposals.

Seamless technology seems the keystone for a future mass production of superconducting resonators. Among all the seamless techniques, only hydroforming and spinning are the ones deserving attention. Both give equivalent results. Therefore, the technique that will be successfully selected for future colliders is only the one that will proof to have the highest reliability, the shortest fabrication time and the lowest cost.

\section{References}

[1] V. Palmieri, "Superconducting resonant cavities", European Training on Technologies and Industr. Appl. of Superconductivity, A. Barone, A. Morini, L. Frunzio eds., World Scientific Publ. pp. 224-255 (1992)

[2] C. Adolphsen et al., "The International Linear Collider Technical Design Report - 3.I: Accelerator R\&D in the Technical Design Phase (2013) http://arxiv.org/pdf/1306.6353v1

[3] http://www.aps.org/units/fip/newsletters/201502/collider.cfm

[4] D. Proch, Cost reduction in cavity fabrication, The 10th Workshop on RF Superconductivity, Tsukuba, Japan (2001) http://accelconf.web.cern.ch/AccelConf/srf01/papers/ fa011-1.pdf 
[5] C. Benvenuti, Superconducting Coatings for Accelerating RF Cavities: Past, Present, Future, Particle Accelerators, 40, pp. $43-57$ (1992)

[6] V. Palmieri et al, "Installation in the LNL ALPI Linac of the First Cryostat with four Niobium sputtered Copper Quarter Wave Resonators", Nucl. Instr. and methods in Phys. Res. A 382, pp. 112-117 (1996)

[7] W. Venturini Delsolaro, S. Calatroni, B. Delaup, A. D’Elia, N. M. Jecklin, Y. Kadi, G. Keppel, D. Lespinasse, P. Maesen, I. Mondino, V. Palmieri, S. Stark, A. R. M. Sublet, M. Therasse, Nb sputtered Quarter Wave Resonators for HIE-ISOLDE, Proceedings of SRF2013, Paris, France, (2014)

[8] W. Singer W. , J. Iversen J., A. Matheisen, H. Weise, P. Michelato, The Challenge and Realization of the Cavity Production and Treatment in Industry for the European XFEL, Proceedings of SRF2013, Paris, France, S.Bousson ed. (2014), http://accelconf.web. cern.ch/AccelConf/SRF2013/papers/moioa03.pdf

[9] T. Fujino, H. Inoue, K. Saito, S. Noguchi, M. Ono, E. Kako, T. Shishido, A. Kubota, S. Koide, "Status of the Seamless L-Band Cavity Fabrication at KEK", Proceedings of the $6^{\text {th }}$ SRF 1995 , Gif-sur-Yvette (France) (1995)

[10] Palmieri V., "Seamless Cavities, the most Creative Topic in rf Superconductivity", Particle Accelerators, 61, pp. 479-519 (1998) http: / /accelconf . web . cern . ch/AccelConf/ SRF97/papers/srf97c18.pdf

[11] H. Kaiser, D. Proch, W. Singer, S. Esin, L. Kravchuk, I. Jelezov, V. Samohvalov, and A. Stepanov, Some technological methods for superconducting cavity manufacturing, Proceedings of the 5th European Particle Accelerator Conference, EPAC'96, Barcelona, Spain, p. 129 (1996)

[12] P. Kneisel, G. Ciovati, X. Singer,W. Singer, and I. Jelezov, Performance of 3-cell seamless niobium cavities, in Proceedings of the 14th SRF2009, Berlin, Germany, 201, pp. 731-734 (2009)

[13] W. Singer, X. Singer, I. Jelezov, P. Kneisel, Hydroforming of elliptical cavities”, Physical Review Special Topics - Accelerators and Beams 18, 022001 (2015)

[14] V. Palmieri, R. Preciso, V.L. Ruzinov, S. Yu.Stark, S. Gambalonga, "Forming Of Seamless Accelerating Resonators Of The Reentrant Type By The Spinning Technique", Nuclear Instruments and methods in physics research A 342, 2/3, pp. 353-356 (1994)

[15] V. Palmieri, R. Preciso, V.L. Ruzinov, S.Yu Stark, L. Badan, I.I. Kulik, "Application of the Spinning Technique to the Production of High Beta Seamless Superconducting Resonators for Particle Accelerators", IEEE Trans. on Applied Superconductivity, 5, No. 2, 315 (1995)

[16] P. Kneisel, V. Pamieri, K. Saito, "Status Of Art Of Spun Cavities", Proceedings of the 9th Workshop on RF Superconductivity, LA-13782-C, November 1-4, 1999 Santa Fè, NM, p. 532 (1999) 